\title{
BMJ
}

\section{Effect of using an interactive booklet about childhood respiratory tract infections in primary care consultations on reconsulting and antibiotic prescribing: a cluster randomised controlled trial}

\author{
Nick A Francis, medical research council health services fellow, ${ }^{1,2}$ Christopher C Butler, professor of primary \\ care medicine, head of department of primary care and public health, ${ }^{1}$ Kerenza Hood, reader in statistics, \\ director of south east Wales trials unit, ${ }^{1,2}$ Sharon Simpson, senior research fellow, ${ }^{1,2}$ Fiona Wood, lecturer, \\ Jacqueline Nuttall, senior trial manager ${ }^{1,2}$
}

\begin{abstract}
${ }^{1}$ Department of Primary Care and Public Health, School of Medicine, Cardiff University, Neuadd Meirionnydd, Heath Park, Cardiff CF14 4XN

${ }^{2}$ South East Wales Trials Unit, School of Medicine, Cardiff University

Correspondence to: N Francis francisna@cf.ac.uk
\end{abstract}

Cite this as: $B M J$ 2009;339:b2885 doi:10.1136/bmj.b2885 Design Pragmatic cluster randomised controlled trial.

\section{ABSTRACT}

Objective To establish whether an interactive booklet on respiratory tract infections in children reduces reconsultation for the same illness episode, reduces antibiotic use, and affects future consulting intentions, while maintaining parental satisfaction with care. Setting 61 general practices in Wales and England. Participants 558 children (6 months to 14 years) presenting to primary care with an acute respiratory tract infection (7 days or less). Children with suspected pneumonia, asthma or a serious concomitant illness, or needing immediate hospital admission were excluded. Three withdrew and 27 were lost to follow-up, leaving 528 (94.6\%) with main outcome data.

Interventions Clinicians in the intervention group were trained in the use of an interactive booklet on respiratory tract infections and asked to use the booklet during consultations with recruited patients (and provide it as a take home resource). Clinicians in the control group conducted their consultations as usual.

Main outcome measures The proportion of children who attended a face-to-face consultation about the same illness during the two week follow-up period. Secondary outcomes included antibiotic prescribing, antibiotic consumption, future consulting intentions, and parental satisfaction, reassurance, and enablement.

Results Reconsultation occurred in $12.9 \%$ of children in the intervention group and $16.2 \%$ in the control group (absolute risk reduction 3.3\%, 95\% confidence interval $-2.7 \%$ to $9.3 \%, P=0.29$ ). Using multilevel modelling (at the practice and individual level) to account for clustering, no significant difference in reconsulting was noted (odds ratio $0.75 ; 0.41$ to 1.38 ). Antibiotics were prescribed at the index consultation to $19.5 \%$ of children in the intervention group and $40.8 \%$ of children in the control group (absolute risk reduction 21.3\%, 95\% confidence interval 13.7 to 28.9), P<0.001). A significant difference was still present after adjusting for clustering (odds ratio
$0.29 ; 0.14$ to 0.60 ). There was also a significant difference in the proportion of parents who said they would consult in the future if their child developed a similar illness (odds ratio $0.34 ; 0.20$ to 0.57 ). Satisfaction, reassurance, and parental enablement scores were not significantly different between the two groups.

Conclusions Use of a booklet on respiratory tract infections in children within primary care consultations led to important reductions in antibiotic prescribing and reduced intention to consult without reducing satisfaction with care.

Trial registration Current Controlled Trials ISRCTN46104365

\section{INTRODUCTION}

Respiratory tract infections are the most common reason for patients to consult in primary care, with children consulting more than any other age group. ${ }^{1}$ One in five children who consult for a respiratory tract infection returns during the same illness episode, and many of these visits are unnecessary. ${ }^{23}$ Unnecessary re-consulting represents an opportunity cost and can increase the pressure on clinicians to prescribe antibiotics. Acute cough in children alone is estimated to cost the NHS at least $£ 31.5 \mathrm{~m}(€ 36.8 \mathrm{~m} ; \$ 51.4 \mathrm{~m})$, with most of this cost arising from consultations with general practitioners. ${ }^{4}$

Complications of respiratory tract infections are rare, and there is little or no benefit from treatment with antibiotics. ${ }^{5-9}$ Guidelines of the National Institute for Health and Clinical Excellence (NICE) recommend against the immediate use of antibiotics for most children who have respiratory tract infections, and promote effective communication and information provision including an indication of the likely duration of illness. ${ }^{10}$ Nevertheless, antibiotics continue to be overprescribed for these illnesses, ${ }^{11}{ }^{12}$ with children receiving more antibiotics than any other age group. ${ }^{13}$ Prescribing for non-specific upper respiratory tract infections, which declined in the late 1990s, is 
once again increasing. ${ }^{14}$ Unnecessary antibiotic use wastes healthcare resources, encourages further consulting in the future for similar illnesses,${ }^{15}$ contributes to the problem of antibiotic resistance, and unnecessarily exposes patients to risk of adverse effects. ${ }^{16}$

Parental beliefs, fears, and expectations play an important part in both consulting behaviour and determining whether an antibiotic is prescribed. ${ }^{17}$ Parents fear serious illness, and worry that they will not be able to recognise the symptoms. ${ }^{18}$ Few are aware of the likely risks and benefits from antibiotic treatment and the normal duration of illness. ${ }^{19}$ Providing information on recognising the signs of serious illness and the likely duration of illness can reduce anxiety, increase confidence, and empower parents to manage their child's illness without needing to consult a healthcare professional. A nurse administered educational intervention aimed at helping parents cope with ear pain in the United States resulted in a reduction in consultations for ear pain over the following year. ${ }^{20}$ Patient information leaflets for adults with lower respiratory tract infections that describe expected duration of illness and suggest simple self-help measures reduce reconsultations ${ }^{21}$ and antibiotic prescribing. ${ }^{22}$

Communication within the consultation is central to addressing parental concerns and expectations, and helps parents to manage their child's illness effectively and safely. Clinicians seldom explicitly ask parents about their expectations about antibiotic treatment, ${ }^{23}$ and overestimate the expectation for antibiotics. ${ }^{24}$ When clinicians believe that patients (and parents) expect antibiotics, they are more likely to prescribe them. ${ }^{17}$ Clinicians often tell parents that their child should recover in a few days, although children usually have symptoms for substantially longer than this. ${ }^{25}$ Setting realistic expectations about the likely duration of illness could reduce parental anxiety and rates of visits. Furthermore, parents value a thorough examination, explanation, reassurance, and advice or guidance more than a prescription for antibiotics. ${ }^{2426}$

We therefore set out to establish whether training clinicians in the use of an interactive booklet, designed to enhance communication within the consultation, and act as a take home resource for parents, would have an effect on rates of reconsultation and antibiotic prescribing. A cluster design was needed, since the intervention was partly directed at the clinicians in the practice. Clinicians who had received training in use of the booklet, and through its use had learnt from its content, would therefore have been unable to not use this knowledge in each consultation where a patient was recruited.

\section{METHODS}

The methods for this cluster randomised controlled trial have been described in detail elsewhere, ${ }^{27}$ and are summarised below.

Half of all general practices from nine local health boards in Wales $(n=147)$ were randomly selected to be sent information about the study (our research group was conducting another randomised controlled trial assessing a related intervention and the other practices were sent information about that study). This procedure was followed by attempts to contact a general practitioner or practice manager in each practice. Telephone contact with a general practitioner or practice manager was successful for 81 practices. Sixty two practices in Wales agreed to take part, although only 49 of these returned a practice agreement and were subsequently randomised. Of the 49 randomised practices, 36 recruited study participants. In England, four primary care research networks agreed to help recruit practices. The total number of practices approached in these networks is not available. However, 38 practices in England verbally agreed to take part, 34 of these returned a practice agreement and were randomised, and 25 of the randomised practices recruited participants. Practices were randomised by a statistician using block randomisation with random block sizes and stratification by practice list size, antibiotic prescribing rate for 2005, and country.

Participating clinicians were asked to recruit sequential eligible children (6 months to 14 years) consulting with a respiratory tract infection (cough, cold, sore throat, earache for seven days or less) and their parents. Exclusions included children with asthma and those with serious ongoing medical conditions such as malignancy or cystic fibrosis.

\section{Sample size calculation}

We calculated that we would need 524 participants recruited from 60 clusters (practices) in order to show a reduction in the proportion of children who reconsult from $20 \%$ to $10 \%$, with $80 \%$ power, at a $5 \%$ significance level, and with an intracluster coefficient of 0.04 . Our aim was to ask 60 practices to recruit ten children each (total of 600 participants) which would allow for loss to follow-up and missing data.

\section{The intervention}

The intervention consisted of an eight page booklet on respiratory tract infections in children, designed to be used within the consultation and then provided to parents as a take home resource (see www.equipstudy. com). Online training on the use of the booklet was also provided for clinicians. The study booklet was developed through a multistage process which has been described elsewhere. ${ }^{28}$ The online training described the content and aims of the booklet, and encouraged its use within the consultation to facilitate the use of certain communication skills, mainly exploring the parent's main concerns, asking about their expectations, and discussing prognosis, treatment options, and any reasons that should prompt reconsultationhttp:///?. Clinicians in practices randomised to the control group were asked to conduct the consultation in their usual manner.

\section{Measures}

Baseline data, including age, duration of illness, and symptoms, were collected by participating clinicians 
at the time of recruitment. We asked clinicians to collect non-identifiable data for all potentially eligible patients (including those who were not approached, those who were approached but were ineligible, and those who declined participation) to assess for possible selection bias. Follow-up was via a telephone administered questionnaire with the child's parent or guardian, 14 days after recruitment. Where a participant's parent could not be contacted on day 14, further attempts were made for at least three days. If still unsuccessful, the telephone number was checked with the relevant general practice and with directory inquiries, and if this approach was unsuccessful then a self completion questionnaire was sent to the parents. Follow-up measurements included reported consultations in primary and secondary care in the two weeks after recruitment, prescriptions for and use of antibiotics, intention to consult with a similar illness in the future, parental enablement (using a modification of the patient enablement instrument $\left.{ }^{29}\right)$, satisfaction with the index consultation, reassurance, and value of any information given to them during the index consultation. Telephone interviewers were blinded to treatment group and were asked to record any subsequent unblinding of allocation (such as a parent talking about receiving a booklet).

Primary outcome was a reconsultation during the two weeks after the index consultation. Antibiotic prescribing, antibiotic consumption, future consulting intentions, parental satisfaction, perception of the usefulness of information received, reassurance, and enablement were secondary outcomes.

\section{Analysis}

Data were analysed using Stata version 9 and MLwiN version 2.10. After checks for missing data and ranges, and double entry of a $10 \%$ sample of the case report

\begin{tabular}{|c|c|}
\hline \multicolumn{2}{|c|}{ Randomised (83 practices) } \\
\hline$\downarrow$ & $\downarrow$ \\
\hline $\begin{array}{l}\text { Allocated to intervention ( } 41 \text { practices): } \\
\text { Recruited patients ( } 30 \text { practices) } \\
\text { ( } n=274 \text { patients, median per practice }=9.5 \text {, } \\
\text { range } 2-25) \\
\text { Did not recruit patients ( } 11 \text { practices) }\end{array}$ & $\begin{array}{l}\text { Allocated to control ( } 42 \text { practices): } \\
\text { Recruited patients ( } 31 \text { practices) } \\
\text { ( } \mathrm{n}=284 \text { patients*, median per practice }=10, \\
\text { range } 1-22) \\
\text { Did not recruit patients (11 practices) }\end{array}$ \\
\hline$\downarrow$ & $\downarrow$ \\
\hline $\begin{array}{l}\text { Lost to follow-up (0 practices) } \\
\text { Patients withdrew }(n=1) \\
\text { Patients lost to follow-up }(n=17)\end{array}$ & $\begin{array}{l}\text { Lost to follow-up (0 practices) } \\
\text { Patients withdrew }(n=2) \\
\text { Patients lost to follow-up }(n=10)\end{array}$ \\
\hline$\downarrow$ & $\downarrow$ \\
\hline $\begin{array}{l}\text { Analysed (30 practices) } \\
\text { Full telephone interviews completed } \\
(n=246,90 \%) \\
\text { Postal questionnaires returned }(n=10,4 \%) \\
\text { Primary outcome data available }(n=256,93 \%)\end{array}$ & $\begin{array}{l}\text { Analysed ( } 31 \text { practices) } \\
\text { Full telephone interviews completed } \\
\text { ( } n=262,93 \%) \\
\text { Partial telephone interview completed } \\
\text { (enablement instrument aborted because of } \\
\text { language problems) }(n=1) \\
\text { Postal questionnaires returned ( } n=9,3 \%) \\
\text { Primary outcome data available }(n=272,96 \%)\end{array}$ \\
\hline
\end{tabular}

Fig 1 | Study profile. *One patient from the control group was subsequently found to have longstanding asthma and was therefore determined (after consultation with the trial steering committee) to have been "recruited in error" and has not been included as a recruited patient forms, Stata was used to obtain summary statistics and undertake univariate analyses. "Satisfaction" and "usefulness of information received" were measured using five point items, but their response distributions were highly skewed. For this reason, these items were transformed into binary outcomes, split into "very satisfied" or "satisfied" versus "neutral", "dissatisfied" or "very dissatisfied" and "very useful" or "useful" versus "neutral", "unhelpful" or "very unhelpful". Similarly, the outcome "reassured" was transformed from a three response item into a binary outcome, split into "very reassured" versus "a little reassured" or "not reassured". The enablement score was calculated in the standard way, but since one item had been excluded, possible scores ranged from 0 to 10 . Enablement scores had a skewed distribution and were therefore converted into a binary outcome using a midrange cutpoint of 5 .

The primary analysis was intention to treat, conducted by fitting two level (practice and patient) random intercept logistic regression models using MLwiN. Similar models were fitted for the secondary outcomes.

After the initial analyses, sensitivity analyses were done by adding the stratifying variables (practice size, practice prescribing status, and country), age, duration of illness, and any symptoms found to be significantly associated in univariate analyses at the $10 \%$ level into each model as covariates. Exploratory analyses were conducted by including factors likely to affect reconsulting and antibiotic prescribing into these two models. The interaction factors were then examined to look for subgroup effects.

\section{RESULTS}

Eighty-three practices were randomised, and 61 of these recruited a total of 558 eligible patients between October 2006 and April 2008 (fig 1).

Intervention and control practices, and randomised practices that did and did not recruit participants, were similar in terms of list size, antibiotic prescribing history, and location (Wales or England) (table 1). Patients recruited by intervention and control practices were similar in terms of age, sex, duration of illness, and symptoms (table 1). Patients were recruited by intervention and control practices at a similar rate (fig 2). We achieved a follow-up rate of 94.6\% (93.4\% intervention, $95.8 \%$ control) for the primary outcome data. Telephone interviewers reported becoming aware of the participant's treatment group in 34 of 509 interviews $(6.7 \%)$.

The number and proportion of patients experiencing each outcome, and odds ratios (with 95\% confidence intervals) for the primary and secondary outcomes are shown in table 2 . There was no significant difference between the intervention and control groups in the odds of reconsulting in primary care during the two weeks after registration. Children in the intervention group were significantly less likely to receive a prescription for antibiotics at the index consultation, less likely to take antibiotics during the first 


\begin{tabular}{|c|c|c|}
\hline & Intervention & Control \\
\hline Randomised, non-recruiting practices & 11 & 11 \\
\hline Median (IQR) list size & 7000 (3680 to 12000$)$ & 8300 (4300 to 9200$)$ \\
\hline No (\%) above average prescribing practice & $5(45.5)$ & $3(27.3)$ \\
\hline No (\%) of practices in England & $3(27.3)$ & $6(54.6)$ \\
\hline Recruiting practices & 30 & 31 \\
\hline Median (IQR) list size & 6750 (4400 to 9000$)$ & 6800 (3700 to 8700$)$ \\
\hline No (\%) above average prescribing & $9(30.0)$ & $10(32.3)$ \\
\hline No (\%) of practices in England & $14(46.7)$ & $11(35.5)$ \\
\hline Median (IQR) cluster size & 9.5 (5 to 10$)$ & $10(7$ to 10$)$ \\
\hline Participating clinicians & 55 & 53 \\
\hline No (\%) of nurses & $5(9.1)$ & $11(20.8)$ \\
\hline Proportion of patients recruited by a nurse & $11.4 \%$ & $19.4 \%$ \\
\hline Patients & 274 & 284 \\
\hline Mean (SD) age (years) & $5.1(3.9)$ & $5.3(3.8)$ \\
\hline Male & $45.3 \%$ & $53.5 \%$ \\
\hline Duration of illness, days (SD) & $3.2(1.7)$ & $3.3(1.8)$ \\
\hline \multicolumn{3}{|l|}{ No (\%) with symptom: } \\
\hline Cough & $173(63.4)$ & $167(58.8)$ \\
\hline Earache & $74(27.1)$ & 69 (24.3) \\
\hline Runny nose & $85(31.1)$ & $97(34.2)$ \\
\hline Sore throat & $89(32.6)$ & $112(39.4)$ \\
\hline Fever & $103(37.7)$ & 109 (38.4) \\
\hline Looks unwell & $36(13.2)$ & 48 (16.9) \\
\hline
\end{tabular}

two weeks, and their parents were less likely to report that they would consult in the future if their child had a similar illness. There were no significant differences in terms of satisfaction, level of reassurance, parental enablement, or the parent's rating of the "usefulness of any information received in the consultation." Similar results were found at the univariate level, with a non-significant difference in reconsulting (absolute risk reduction 3.3\%, 95\% confidence interval $-2.7 \%$ to $9.3 \%, \mathrm{P}=0.29$ ), and significant differences in antibiotic prescribing (absolute risk reduction 21.3\%, number needed to treat $4.7, \mathrm{P}<0.001$ ), antibiotic consumption (absolute risk reduction 20.6\%, 95\% confidence interval $12.7 \%$ to $28.5 \%$, number needed to treat $4.9, \mathrm{P}<0.001)$, and future consulting intentions $(21.1 \%, 13.1 \%$ to $29.2 \%, 4.7, \mathrm{P}<0.001)$.

There was no significant intervention effect when telephone consultations were counted as reconsultations along with face to face primary care consultations (odds ratio $0.81 ; 0.47$ to 1.42 ), or when consultations at accident and emergency departments were included along with primary care consultations $(0.85 ; 0.48$ to 1.51). The intervention had a similar effect size on the antibiotic outcomes of receiving a prescription for antibiotics for immediate use at the index consultation (excluding prescriptions for delayed use; $0.26 ; 0.11$ to 0.62 ) and receiving an antibiotic prescription at any point in the two week follow -up $(0.31 ; 0.16$ to 0.62$)$.

The sensitivity analyses for the main reconsultation outcome and the antibiotic prescribing outcome did not result in any meaningful changes to the results (that is, there were no significant intervention effects in the sensitivity analyses for the reconsultation outcome and similar significant effects for all analyses with the antibiotic prescribing outcome).

\section{Subgroup analyses}

No significant interaction effects were seen in the reconsultation models. In the antibiotic prescribing model, the intervention was more effective in aboveaverage prescribing practices (table 3 ). There were no other significant interaction effects.

\section{Adverse events}

Seven patients (three in the intervention group and four in the control group) were subsequently admitted to hospital or observed in a paediatric assessment unit. One patient in the control group had a longstanding diagnosis of asthma, and as such was excluded (after discussion in the trial steering committee). The longest hospital admission (two nights) was a patient in the intervention group who had febrile convulsions. The remaining admissions were one night or less.

\section{Comparing recruited and non-recruited patients}

Ninety three patients were not recruited into the study (50 were ineligible, 27 declined participation, and 16 were not recruited because of a lack of time in the consultation or for other unspecified reasons. There were no significant differences between recruited and nonrecruited patients in terms of age or presenting symptoms; however, cough was more common in nonrecruited patients $(71.9 \% \quad v 61.0 \%)$. Of the nonrecruited patients, there was no significant difference in cough between the intervention and control groups. Previous duration of illness was higher in nonrecruited patients than in recruited patients, although this finding was not surprising, since a duration of illness of more than seven days was a common reason for exclusion from the study.

\section{DISCUSSION}

Clinicians' use of an interactive booklet on respiratory tract infections in children within primary care consultations resulted in a significant reduction in antibiotic prescribing and consumption and high levels of parental satisfaction. Use of the intervention did not result in a

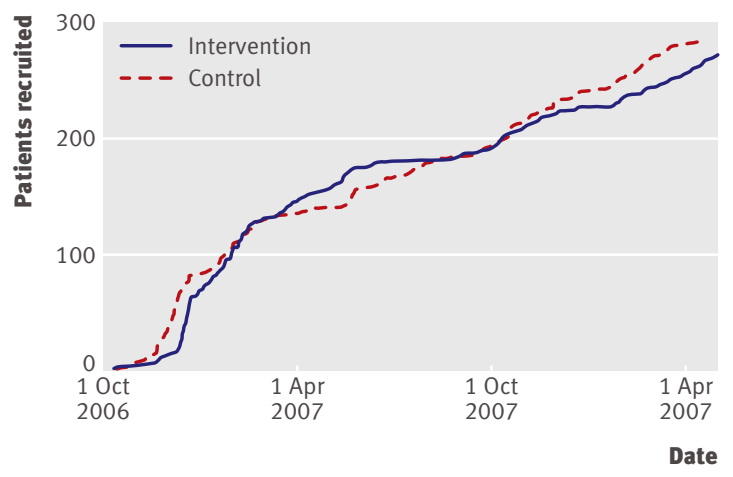

Fig $2 \mid$ Recruitment rates in the two groups 
Table 2 |Effect of the intervention on patient outcomes

\begin{tabular}{|c|c|c|c|}
\hline & \multicolumn{2}{|c|}{$\begin{array}{l}\text { No (\%) experiencing } \\
\text { the outcome }\end{array}$} & \multirow{2}{*}{$\begin{array}{l}\text { Odds ratio from } \\
\text { multilevel modelling } \\
(95 \% \mathrm{Cl})\end{array}$} \\
\hline & Intervention & Control & \\
\hline \multicolumn{4}{|l|}{ Outcomes with data collected from telephone administered and postal questionnaires } \\
\hline No of patients & 256 & 272 & \\
\hline $\begin{array}{l}\text { Primary outcome: primary care reconsultation within first two weeks* } \\
\text { (intracluster correlation coefficient }=0.06 \text { ) }\end{array}$ & $33(12.9)$ & $44(16.2)$ & 0.75 (0.41 to 1.38$)$ \\
\hline Antibiotic prescribed at index consultation (intracluster correlation coefficient=0.24) & $50(19.5)$ & $111(40.8)$ & $0.29(0.14$ to 0.60$)$ \\
\hline \multicolumn{4}{|l|}{ Outcomes with data collected from telephone administered questionnaire only } \\
\hline No of patients & 246 & 263 & \\
\hline $\begin{array}{l}\text { Antibiotics taken within first two weeks (including antibiotics prescribed } \\
\text { after index consultation) }\end{array}$ & $55(22.4)$ & $111(43.0)$ & $0.35(0.18$ to 0.66$)$ \\
\hline Parent intends to consult if their child has similar illness in future & $136(55.3)$ & $201(76.4)$ & $0.34(0.20$ to 0.57$)$ \\
\hline Parental enablement score $(\geq 5)$ & $99(40.2)$ & $94 †(35.9)$ & $1.20(0.84$ to 1.73$)$ \\
\hline Satisfaction $\ddagger$ & $222(90.2)$ & $246(93.5)$ & 0.64 (0.33 to 1.22$)$ \\
\hline Reassurance§ & $177(72.0)$ & $198(75.3)$ & $0.84(0.57$ to 1.25$)$ \\
\hline Usefulness of information received $\mathbb{T}$ & $210(85.4)$ & $224(85.2)$ & 1.01 (0.60 to 1.68$)$ \\
\hline \multicolumn{4}{|c|}{$\begin{array}{l}\text { *Parental report that child attended a face to face consultation with a primary care clinician in their general practice, or with an out-of-hours provider, } \\
\text { in the two weeks after registration. } \\
\text { †No=262 for this group as one parent was unable to complete enablement questions because of language problems. } \\
\ddagger \text { Proportion of parents who reported being very satisfied or satisfied with the consultation. } \\
\S \text { Proportion of parents who reported feeling very reassured after their consultation. } \\
\text { \Proportion of parents who reported that information they received in the consultation was very useful or useful. }\end{array}$} \\
\hline
\end{tabular}

significant reduction in the proportion of children who reconsulted in the two weeks after the index consultation. However, fewer parents in the intervention group said that they would consult in the future should their child develop a similar illness. No significant differences were seen in terms of parental satisfaction, reassurance, enablement, or perception of the usefulness of any information received about their child's illness.

\section{Strengths and limitations of the study}

This was an adequately powered randomised controlled trial. Practices were recruited from throughout Wales and England and were broadly representative of UK general practice. The results of this study are therefore likely to be highly generalisable to UK general practice. The stratified randomisation procedure helped ensure practices in both groups were similar in terms of size, location, and historical antibiotic prescribing rate. We achieved the target sample for both clusters (general practices) and patients, with a high follow-up rate.

Cluster randomised designs can increase risk of selection bias. Our intervention was aimed not only at individual patients, but also at the primary care clinicians (through the online training). For this reason, an individually randomised trial was not possible: once trained in new consulting skills, clinicians cannot switch back to their untrained state. Selection bias can occur at the level of the cluster (that is, through differential dropout) or the individual. There were 11 practices in each arm of the trial that did not recruit any participants. Of these 22 practices, there were no significant differences in terms of list size, historical prescribing rate, or proportion located in Wales or England. We asked all participating clinicians to identify sequential eligible patients, and to record non- identifiable data for all those who were not recruited, in order to look for evidence of selection bias at the individual level. We found no important differences in the patients who were and were not recruited or between the patients who were not recruited by clinicians in the intervention and control groups. Similar recruitment rates in the two groups also suggest minimal selection bias.

The non-significant difference in scores of parental enablement and usefulness of information received are surprising and seem inconsistent with the significant reduction in the proportion of parents stating that they would consult with a future similar illness. The patient enablement instrument was designed for first person use in routine general practice consultations and might not have been sensitive enough for measuring changes in parental enablement two weeks after the consultation.

Clinicians in the control group might have altered their behaviour (towards providing more information than usual) as a result of their participation in the study, which could have attenuated any effect that changes in the behaviour of doctors in intervention practices might have had on parental satisfaction, enablement, and usefulness of information received. We are exploring the effects of the intervention on parental knowledge and beliefs in a qualitative process evaluation.

Neither clinicians nor participants were blinded as to study group. As our intervention was directed partly at clinicians, a change in their behaviour was both expected and desirable. However, we need to distinguish between changes related to use of the intervention and changes associated with an awareness of being observed (Hawthorne effect). All participating clinicians were provided with information about the aims of the study. However, antibiotic use was listed 
fourth in a long list of outcome measures and is therefore unlikely to have resulted in meaningful changes in prescribing behaviour. The effect of the intervention on antibiotic prescribing was not modified by practice location, which, given that many practices in Wales would be aware of the research group's interest in antibiotic prescribing, suggests that the Hawthorne effect was unlikely to be playing a significant part. Clinicians did not have any involvement in measuring outcomes, and are therefore unlikely to have contributed to ascertainment bias. Children (and their parents) were not blinded to treatment group. However, practices were instructed not to inform participants of the group to which they had been assigned before obtaining consent. Telephone interviewers were blinded to trial group in $93 \%$ of all interviews.

Although we recognise that interventions are not always delivered as planned in pragmatic trials, we did not measure treatment fidelity because we wanted the assessors to remain blinded to study group where possible. However, suboptimum fidelity of intervention delivery is likely to dilute the treatment effect and therefore could have led to a type II error regarding reconsultations, but is unlikely to have led to a type I error regarding the positive findings.

There were unequal numbers of nurses in the study groups (and thus patients recruited by nurses). We believe that this discrepancy was due to chance. We found no association between clinician's profession (doctor or nurse) and either reconsultations or antibiotic prescribing, either at a univariate level or using multilevel modelling, and therefore believe that this did not have an important effect on our results.

\section{Comparison with other published work}

Our findings are consistent with those of Macfarlane and colleagues who found that the use of a leaflet on lower respiratory tract infection in adults resulted in a reduction in antibiotic use by nearly $25 \% .{ }^{22}$ However, Macfarlane and colleagues have also shown a reduction in reconsultations from use of a leaflet, ${ }^{21}$ whereas our results did not show a significant reduction. This finding might be because the underlying reconsultation rate in our study was lower than that found by Macfarlane and lower than that used in our sample size calculation. This lower rate could indicate societal changes in knowledge or beliefs over time and might be much closer to a desirable level of reconsulting, and therefore more difficult to reduce. Certainly the 3.3\% absolute difference found in our study was

Table 3 |Effect of practice prescribing history and study intervention on probability of being prescribed an antibiotic

\begin{tabular}{lcc} 
& \multicolumn{2}{c}{ Practice antibiotic prescribing history } \\
\cline { 2 - 3 } & $\begin{array}{c}\text { Higher (above national } \\
\text { average for 2005) }\end{array}$ & $\begin{array}{c}\text { Lower (below national } \\
\text { average for 2005) }\end{array}$ \\
\hline Intervention & $16.3 \%$ & $15.4 \%$ \\
\hline Control & $64.1 \%$ & $27.3 \%$ \\
\hline
\end{tabular}

Values show probability of being prescribed an antibiotic, calculated from coefficients derived from multilevel modelling. substantially smaller than the $10 \%$ reduction we had considered to be clinically important. Although we did not identify any studies that used a booklet designed specifically for use in consultations on respiratory tract infections in children, studies that have evaluated sending information booklets on minor illnesses to patients' homes have generally found little effect on consultation rates. ${ }^{30-34}$ A recent study in the United States found that a sustained, multifaceted intervention, conducted over three years and aimed at reducing antibiotic prescribing in young children, resulted in minimal reduction in antibiotic use beyond underlying trends. ${ }^{35}$ The intervention in this study included several printed and web-based educational materials but did not encourage interactive use of the material within the consultation. Use of a leaflet for patients with lower respiratory tract infections resulted in an increase in reconsultations in the first month, and no significant difference in use of antibiotics or satisfaction. ${ }^{36}$ However, the leaflet in this study was brief, was not designed for interactive use in the consultation, and was provided in addition to verbal information about the natural history. A further United States study found that providing patients with a pack containing a pamphlet, a sticker, and a thermometer was associated with reduced consultation rates. However, this study was limited by non-random allocation and post allocation exclusion of patients. ${ }^{37}$ Another study where parents were randomised to receive written materials on either antibiotic use or injury prevention found no reduction in antibiotic use in the families who received the intervention. ${ }^{38}$ This finding could indicate the need to provide parents with positive messages (how best to manage the illness) rather than negative ones (don't use antibiotics).

\section{Interpretation of the results}

We found a statistically non-significant reduction in the proportion of children who reconsulted in the intervention group, which was considerably smaller than the $10 \%$ difference that was specified as clinically meaningful.

We did demonstrate statistically and clinically significant reductions in antibiotic prescribing and consumption, which have important implications for policy makers, practitioners, and ultimately patients. How the reduction in prescribing was mediated is not yet clear, but it was possibly through a combined effect on clinician and parental behaviour. Clinicians probably recognised the importance of changing their prescribing behaviour and felt that they had the resources to effectively achieve this.

A significant reduction in the proportion of parents who said that they would consult if their child had a similar illness in the future is encouraging and suggests that use of the intervention could have an effect on future consulting.

No differences were recorded in terms of satisfaction, reassurance, value of information received, or parental enablement. Reassuringly, a high level of satisfaction 


\section{WHAT IS ALREADY KNOWN ON THIS TOPIC}

Respiratory tract infections in children are largely self limiting and benefit very little from antibiotic treatment. However, consultation rates continue to be high and antibiotics are still frequently prescribed.

\section{WHAT THIS STUDY ADDS}

Providing primary care clinicians with a carefully developed booklet on respiratory tract infections in children, and training in its use within the consultation, reduces antibiotic prescribing by around two thirds. Satisfaction among parents receiving this intervention was high, and no significant difference was found between those receiving the intervention and those receiving usual care.

Use of this intervention seems to have little effect on reconsulting for the same illness episode, but does reduce future consulting intentions.

Clinicians should consider the use of this intervention in routine consultations with children with respiratory tract infections. was reported in the intervention group despite the significant reduction in antibiotic prescribing.

The routine use of this intervention in primary care should now be considered along with other effective interventions such as delayed prescribing. ${ }^{39}$ The magnitude of the reduction in antibiotic prescribing achieved suggests that its use could have important implications for patients, and, as a result of the threat posed by increasing antimicrobial resistance, for public health. Furthermore, the booklet and online training could be produced and distributed fairly cheaply. Its use also seems to be safe and result in high levels of parental satisfaction. However, like any complex intervention, the precise elements that contributed to its effectiveness are unclear. The intervention not only provided parents with a take-home resource, it also aimed to modify the consultation process (especially communication within the consultation), which could have had an effect on consultation length. We are currently exploring which aspects of the intervention led to its effectiveness, the impact of its use on consultation length, its effects on long term consulting rates, and its economic impact. For example, we do not know if another booklet or leaflet on the same subject would result in a similar effect, or whether the training programme or the interactive use of the booklet was important. In the meantime, higher prescribing clinicians, or those who would like to reduce their prescribing but feel that they lack the tools to achieve this, might wish to consider use of this intervention.

We are grateful to all the patients, parents, and clinicians who participated in the development of the intervention and the trial. We gratefully acknowledge the support received from the Primary Care Research Network and from all participating research networks. We thank the administrative staff in the Department of Primary Care and Public Health and the South-East Wales Trials Unit who worked hard to ensure the success of the study. We also gratefully acknowledge the contribution made by members of the independent trial steering committee. Contributors: CB conceived the study. NF, FW, CB, KH, and SS developed the intervention. $\mathrm{CB}, \mathrm{NF}, \mathrm{KH}$, and SS wrote the protocol. All contributors sat on the study management group. NF managed the trial and NF and JN conducted the telephone interviews. NF wrote the first draft of the paper and all authors made subsequent contributions. NF is the guarantor. Funding: We gratefully acknowledge funding from the Medical Research Council and the Welsh Assembly Government in the form of a joint Health Services Fellowship for NF. Funding for the development of the training website was from an educational grant from Pfizer UK. The South-East Wales Trials Unit is funded by the Welsh Office for Research and Development. All authors declare that this work was conducted independently from the study funders.

Sponsorship: This study was sponsored by Cardiff University. Competing interests: None declared.

Ethical approval: This study was approved by the South East Wales Local Research Ethics Committee (Reference number 04/WSE04/109).

1 McCormick A, Fleming D, Charlton J. Morbidity statistics from general practice. Fourth national study 1991-1992. London: HMSO, 1995.

2 Butler CC, Robling M, Prout H, Hood K, Kinnersley P. Management of suspected acute viral upper respiratory tract infection in children with intranasal sodium cromoglicate: a randomised controlled trial. Lancet 2002;359:2153-8.

3 Stott NC. Management and outcome of winter upper respiratory tract infections in children aged 0-9 years. BMJ 1979;1:29-31.

4 Hollinghurst S, Gorst C, Fahey T, Hay AD, Hollinghurst S, Gorst C, et al. Measuring the financial burden of acute cough in pre-school children: a cost of illness study. BMC Family Practice 2008;9:10.

5 Arroll B, Kenealy T. Antibiotics for the common cold and acute purulent rhinitis. Cochrane Database Syst Rev 2005;3:CD000247.

6 Del Mar C, Glasziou PP, Spinks A. Antibiotics for sore throat. Cochrane Database Syst Rev 2006;4:CD000023.

7 Smith SM, Fahey T, Smucny J, Becker Lorne A. Antibiotics for acute bronchitis. Cochrane Database Syst Rev 2004;4:CD000245.

8 Spurling GKP, Fonseka K, Doust J, Del Mar C. Antibiotics for bronchiolitis in children. Cochrane Database Syst Rev 2007;1:CD005189.

9 Glasziou PP, Del Mar C, Sanders S, Hayem M. Antibiotics for acute otitis media in children. Cochrane Database Syst Rev 2004;1:CD000219.

10 National Institute for Health and Clinical Excellence. Prescribing of antibiotics for self-limiting respiratory tract infections in adults and children in primary care (Clinical guideline 69). www.nice.org.uk/ CG692008.

11 Ashworth M, Charlton J, Cox K, Gulliford M, Latinovic R, Rowlands G Why has antibiotic prescribing for respiratory illness declined in primary care? A longitudinal study using the General Practice Research Database. J Public Health 2004;26:268-74.

12 Shapiro E. Injudicious antibiotic use: an unforeseen consequence of the emphasis on patient satisfaction? Clin Ther 2002;24:197-204.

13 Akkerman AE, van der Wouden JC, Kuyvenhoven MM, Dieleman JP, Verheij TJM. Antibiotic prescribing for respiratory tract infections in Dutch primary care in relation to patient age clinical entities. J Antimicrob Chemother 2004;54:1116-21.

14 Thompson PL, Spyridis N, Sharland M, Gilbert RE, Saxena S, Long PF, et al. Changes in clinical indications for community antibiotic prescribing for children in the UK from 1996-2006: will the new NICE prescribing guidance on upper respiratory tract infections be ignored? Arch Dis Child 2008; doi:10.1136/adc.2008.147579.

15 Little P, Gould C, Williamson I, Warner G, Gantley M, Kinmonth AL. Reattendance and complications in a randomised trial of prescribing strategies for sore throat: the medicalising effect of prescribing antibiotics. BM/ 1997;315:350-2.

16 Butler CC, Hillier S, Roberts Z, Dunstan F, Howard A, Palmer S. Antibiotic-resistant infections in primary care are symptomatic for longer and increase workload: outcomes for patients with E. coli UTIs. BrJ Gen Pract 2006;56:686-92.

17 Cockburn J, Pit S. Prescribing behaviour in clinical practice: patients expectations and doctors' perceptions of patients' expectations-a questionnaire study. BMJ 1997;315:520-3.

18 Kai J. What worries parents when their preschool children are acutely ill, and why: a qualitative study. BMJ 1996;313:983-6.

19 Braun BL, Fowles JB. Characteristics and experiences of parents and adults who want antibiotics for cold symptoms. Arch Fam Med 2000;9:589-95

20 McWilliams DB, Jacobson RM, Van Houten HK, Naessens JM, Ytterberg KL. A program of anticipatory guidance for the prevention of emergency department visits for ear pain. Arch Pediatr Adolesc Med 2008;162:151-6.

21 Macfarlane JT, Holmes WF, Macfarlane RM. Reducing reconsultations for acute lower respiratory tract illness with an information leaflet: A randomized controlled study of patients in primary care. $\mathrm{Br} / \mathrm{Gen}$ Pract 1997:47:719-22.

22 Macfarlane J, Holmes W, Gard P, Thornhill D, Macfarlane R, Hubbard R. Reducing antibiotic use for acute bronchitis in primary care: blinded, randomised controlled trial of patient information leaflet. BMJ 2002;324:91-4.

23 Butler CC, Rollnick S, Kinnersley P, Jones A, Stott N. Reducing antibiotics for respiratory tract symptoms in primary care: consolidating 'why' and considering 'how'. Br J Gen Pract 1998;48:1865-70. 
24 Butler CC, Rollnick S, Pill R, Maggs-Rapport F, Stott N. Understanding the culture of prescribing: Qualitative study of general practitioners' and patients' perceptions of antibiotics for sore throats. $B M$ J 1998;317:637-42.

25 Butler CC, Kinnersley P, Hood K, Robling M, Prout H, Rollnick S, et al. Clinical course of acute infection of the upper respiratory tract in children: cohort study. BMJ 2003;327:1088-9.

26 Kallestrup P, Bro F. Parents' beliefs and expectations when presenting with a febrile child at an out-of-hours general practice clinic. Br J Gen Pract 2003;53:43-4.

27 Francis N, Hood K, Simpson S, Wood F, Nuttall J, Butler C. The effect of using an interactive booklet on childhood respiratory tract infections in consultations: study protocol for a cluster randomised controlled trial in primary care. BMC Family Practice 2008;9:23.

28 Francis N, Wood F, Simpson S, Hood K, Butler CC. Developing an 'interactive' booklet on respiratory tract infections in children for use in primary care consultations. Patient Educ Couns 2008;73:286-93.

29 Howie J, Heaney D, Maxwell M, Walker J. A Comparison of a Patient Enablement Instrument (PEI) against two established satisfaction scales as an outcome measure of primary care consultations. Fam Pract 1998;15:165-71.

30 Morrell DC, Avery AJ, Watkins CJ. Management of minor illness. BMJ 1980;280:769-71.

31 Hansen BW. A randomized controlled trial on the effect of an information booklet for young families in Denmark. Patient Educ Couns 1990;16:147-50.
32 Usherwood TP. Development and randomized controlled trial of a booklet of advice for parents. Br J Gen Pract 1991;41:58-62.

33 Heaney D, Wyke S, Wilson P, Elton R, Rutledge P, Sommenville A, et al. Assessment of impact of information booklets on use of healthcare services: randomised controlled trial. BMJ 2001;322:1-5.

34 Little P, Somenille I, Williamson I, Warner G, Moore M, Wiles R, et al. Randomised controlled trial of self management leaflets and booklets for minor illness provided by post. BMJ 2001;322:1214-7.

35 Finkelstein JA, Huang SS, Kleinman K, Rifas-Shiman SL, Stille CJ, Daniel J, et al. Impact of a 16-community trial to promote judicious antibiotic use in Massachusetts. Pediatrics 2008;121:e15-23.

36 Little P, Rumsby K, Kelly J, Watson L, Moore M, Warner G, et al. Information leaflet and antibiotic prescribing strategies for acute lower respiratory tract infection: a randomized controlled trial. JAMA 2005;293:3029-35.

37 Roberts CR, Imrey PB, Turner JD, Hosokawa MC, Alster JM. Reducing physician visits for colds through consumer education. JAMA 1983;250:1986-9.

38 Taylor JA, Kwan-Gett TSC, McMahon EM, Jr. Effectiveness of a parental educational intervention in reducing antibiotic use in children: a randomized controlled trial. Pediatr Infect Dis J 2005;24:489-93.

39 Spurling GKP, Del Mar C, Dooley L, Foxlee R. Delayed antibiotics fo respiratory infections. Cochrane Database Syst Rev 2007;3:CD004417.

Accepted: 4 March 2009 